\title{
Tumor venéreo transmissível canino com metástase encefálica
}

\section{Canine transmissible venereal tumor with encephalic metastasis}

\author{
Ciciane Pereira Marten Fernandes ${ }^{1 *}$; Luiz Fernando Jantzen Gaspar²; \\ Ana Raquel Mano Meinerz²; Fabiane Borelli Grecco3 ${ }^{3}$ Márcia de Oliveira Nobre²; \\ Marlete Brum Cleff ${ }^{2}$
}

\begin{abstract}
Resumo
Relata-se o primeiro caso no Brasil de tumor venéreo transmissível (TVT) vaginal com metástase para o sistema nervoso central (SNC), além do envolvimento do tecido cutâneo, ocular e pulmonar e descreve a resistência do TVT ao tratamento com vincristina. O TVT é uma neoplasia contagiosa ocorrendo, principalmente, em locais em que não ocorre um controle populacional de cães, e atualmente tem sido demonstrada resistência destas células tumorais ao tratamento convencional com vincristina. Foi atendida uma fêmea canina, sem raça definida, com cerca de cinco anos de idade, pesando 9,2kg, com histórico de sangramento vaginal há mais de seis meses. Após diagnóstico citológico de TVT foi recomendado tratamento com quimioterápico. Houve resistência a quimioterapia com sulfato de vincristina, preconizando a utilização de quimioterápico doxorrubicina. Suspeita-se que o desenvolvimento do tumor no sistema nervoso central possivelmente tenha ocorrido devido à doxorrubicina não ter capacidade de atravessar a barreira hematoencefálica, aliado à resistência à vincristina.
\end{abstract}

Palavras-chave: Cão, tumor venéreo transmissível, metástases, encéfalo, doxorrubicina

\begin{abstract}
The first case in Brazil of vaginal TVT with metastasis for the central nervous system is reported, as well as the presence of cutaneous, eye and lung metastases and describes the resistance of the TVT to treatment with vincristine. The TVT is a contagious neoplasm that occurs mainly in places where there is no dog population control, and currently, resistance of these tumor cells to conventional treatment with vincristine has been demonstrated. A mongrel female dog of about five years old, weighing $9.2 \mathrm{~kg}$, with a history of vaginal bleeding for more than six months was attended. After cytological diagnosis, treatment with chemotherapy was recommended. There was resistance to chemotherapy treatment with vincristine sulfate, advocating the use of doxorubicin. It is suspected that tumor development in the central nervous system possibly occurred because the doxorubicin was not capable of crossing the hematoencephalic barrier, along with resistance to vincristine.
\end{abstract}

Key words: Dog, transmissible venereal tumor, metastases, encephalon, doxorubicin

\footnotetext{
${ }^{1}$ Discente de Doutorado em Ciência Animal, Universidade Federal de Pelotas, UFPEL, Pelotas, RS. E-mail: cici.marten@gmail.com

${ }^{2}$ Profs. Drs. do Dept ${ }^{0}$ de Clínicas Veterinária, UFPEL, Pelotas, RS. E-mail: 1fjgaspar@ibest.com.br; rmeinerz@bol.com.br; marciaonobre@gmail.com; emebrum@bol.com.br

${ }^{3}$ Prof $^{a}$ Dr $^{a}$ do Dept ${ }^{0}$ de Patologia Animal, UFPEL, Pelotas, RS. E-mail: fabigrecco@ig.com.br

* Autor para correspondência
} 


\section{Introdução}

O Tumor Venéreo Transmissível (TVT) é uma neoplasia contagiosa que ocorre principalmente na genitália externa de cães (AMARAL et al., 2007; DALECK et al., 2008), podendo acometer pele e cavidade nasal (PARK et al., 2006), olhos (RODRIGUES; ALESSI; LAUS, 2001) e mais raramente o sistema nervoso central (FERREIRA et al., 2000). A casuística de TVT é alta, principalmente em locais em que não ocorre um controle populacional de cães, e atualmente tem sido demonstrada resistência destas células tumorais ao tratamento convencional com vincristina (SILVA et al., 2007; FILGUEIRA, 2010), fato preocupante, já que a quimioterapia com sulfato de vincristina é o tratamento de eleição para TVT (CHABNER et al., 2001; DALECK et al., 2008; SCARPELLI; VALLADÃO; METZE, 2010) e/ou associação com doxorrubicina (GARCEZ et al., 2010; SCARPELLI; VALLADÃO; METZE., 2010). Assim, o objetivo deste trabalho foi relatar o primeiro caso no Brasil de tumor venéreo transmissível vaginal com metástase para o sistema nervoso central, além do envolvimento do tecido cutâneo e pulmonar, e descreve a resistência do TVT ao tratamento com vincristina.

\section{Casuística}

Foi atendida uma fêmea canina, sem raça definida, com cerca de cinco anos de idade, pesando 9,2kg, com histórico de sangramento vaginal há mais de seis meses. No exame clínico foi observado mucosas pálidas, desidratação $5 \%$, tempo de preenchimento capilar acima de dois segundos, temperatura corporal de $38,6^{\circ} \mathrm{C}$, taquicardia (134 bpm) e taquipnéia (45mrm). O paciente apresentava sete nódulos cutâneos, sendo cinco localizados na região abdominal, próximo às mamas abdominais craniais e caudais e dois nódulos na área de mandíbula e um ocular. Todos os nódulos apresentavam consistência firme, não aderidos, com tamanho variando de 1 a $3 \mathrm{~cm}$ de diâmetro, não sendo observado linfadenopatia. $\mathrm{Na}$ avaliação vulvar foi observado aumento de volume com presença de massa nodular (12 cm de comprimento, $14 \mathrm{~cm}$ de largura e $8,2 \mathrm{~cm}$ de espessura), com aspecto de couve-flor, friável e secreção serosanguinolenta, o que permitiu a suspeita clínica de TVT vaginal (Figura 1).

Figura 1. Paciente apresentando nódulos cutâneos e massa nodular com aspecto de couve-flor, friável e secreção serosanguinolenta.

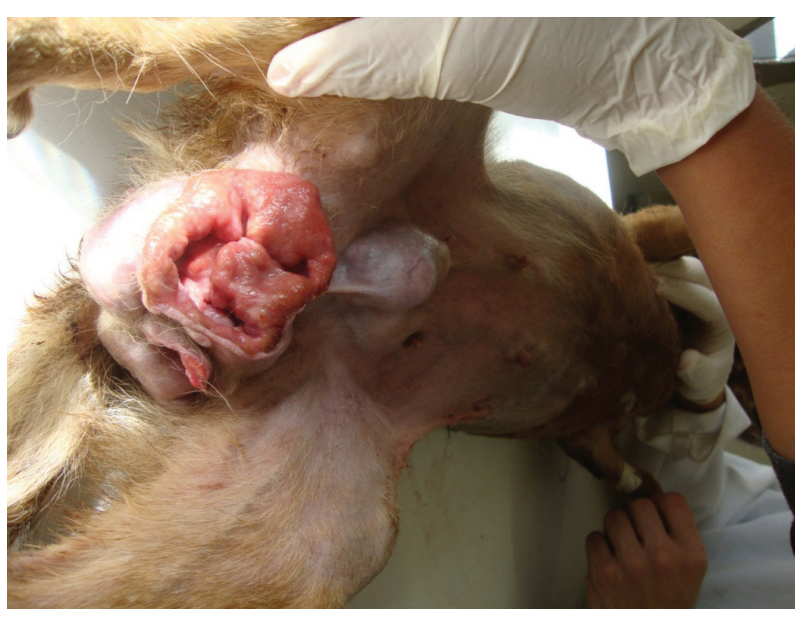

Fonte: Elaboração dos autores.

Foram coletadas amostras do tumor localizado na vulva pelo método de impressão e realizada citologia aspirativa por agulha fina (CAAF) dos nódulos cutâneos. Tanto nas amostras obtidas do tumor vulvar,quanto dos nódulos cutâneos, foram observadas células arredondadas, com núcleo grande, basofílico e presença de vacúolos citoplasmáticos, sendo estas alterações compatíveis com padrão de TVT plasmocitóide.

Para avaliação do estado geral do paciente foi realizado exame hematológico e dosagens séricas de fosfatase alcalina (FA), uréia, creatinina, alaninaminotrasnferase (ALT), aspartatoaminotransferase (AST) e radiografia tóracica. $\mathrm{Na}$ hematologia observou-se anemia regenerativa (hematócrito 36,7\%) e leucocitose $(19,800 \mu \mathrm{L})$ com desvio a esquerda (segmentados 
$16,038 \mu \mathrm{L}$ e bastonetes $396 \mu \mathrm{L})$. Os valores da bioquímica sanguínea assim como, o exame radiográfico torácico, apresentaram-se dentro dos padrões fisiológicos para a espécie.

A partir destes resultados foi preconizado ceftriaxona $(25 \mathrm{mg} / \mathrm{kg})$ de 12-12 horas por sete dias e iniciou-se o tratamento específico do TVT, utilizando sulfato de vincristina $\left(0,5 \mathrm{mg} / \mathrm{m}^{2} \mathrm{IV}\right)$ com intervalos semanais, durante quatro semanas. Foram realizados hemogramas seriados antes de cada aplicação. Após quatro aplicações observouse pequena redução (30\%) da massa tumoral vulvar e praticamente nenhuma involução dos nódulos cutâneos, decidindo-se pela utilização de doxorrubicina $\left(30 \mathrm{mg} / \mathrm{m}^{2}\right.$, IV), em duas aplicações com intervalos de 21 dias.

Com o uso da doxorrubicina foi observada redução importante da massa vulvar na semana seguinte a primeira administração e redução total após a segunda aplicação, com regressão das tumorações cutâneas, entretanto a paciente apresentou anemia arregenerativa $(26,6 \%)$ e leucopenia $(4,300 \mu \mathrm{L})$, além de acentuada perda de peso.

Após 20 dias da aplicação da segunda dose de doxorrubicina, a fêmea canina demonstrou mudança de comportamento, com prostração, deambulação, cegueira, opistótono e convulsões, determinando alterações no sistema nervoso central. Após uma semana do início dos sinais neurológicos o animal foi a óbito sendo encaminhado para realização de necropsia.

Na necropsia foram observadas massas cutâneas com $2 \mathrm{~cm}$ de diâmetro, esbranquiçadas e friáveis próximas às mamas abdominais lado direito, além de duas massas de aproximadamente $3 \mathrm{~cm}$ de diâmetro no lobo pulmonar diafragmático esquerdo. No sistema nervoso central (SNC) notou-se aumento de volume e amolecimento em área do tálamo e em colículo rostral direito (Figura 2).

Figura 2. Corte transversal do encéfalo-região do tálamo da fêmea canina com TVT. Assimetria dos hemisférios cerebrais decorrente de compressão pela massa tumor.

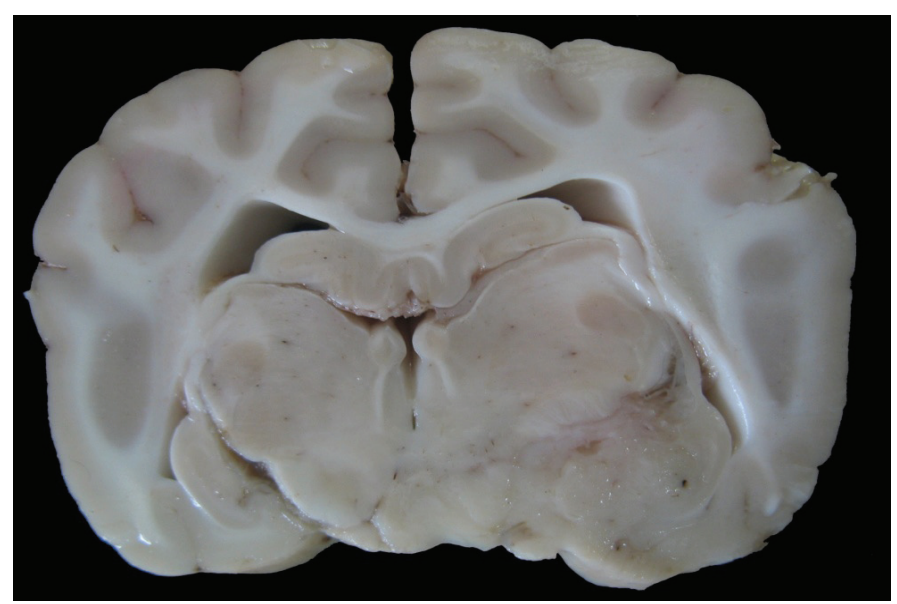

Fonte: Elaboração dos autores.

Essas alterações tornaram-se mais evidentes após a fixação do material em formalina $10 \%$ por um período de 48 horas. As amostras teciduais foram incluídas em parafina, seccionadas em 5 micras e coradas por hematoxilina e eosina. A análise histopatológica das massas cutâneas e pulmonares, bem como dos cortes de SNC, revelou neoplasma constituído por células redondas, normomórficas, com mitoses evidentes, apoiadas sobre delicado e escasso tecido conjuntivo fibroso, desta forma 
confirmando o diagnóstico de TVT cutâneo, pulmonar e no SNC (Figura 3).

Figura 3. Células neoplásicas (seta) limitando e invadindo região da cápsula interna do $\mathrm{SNC}$ da fêmea canina com TVT.

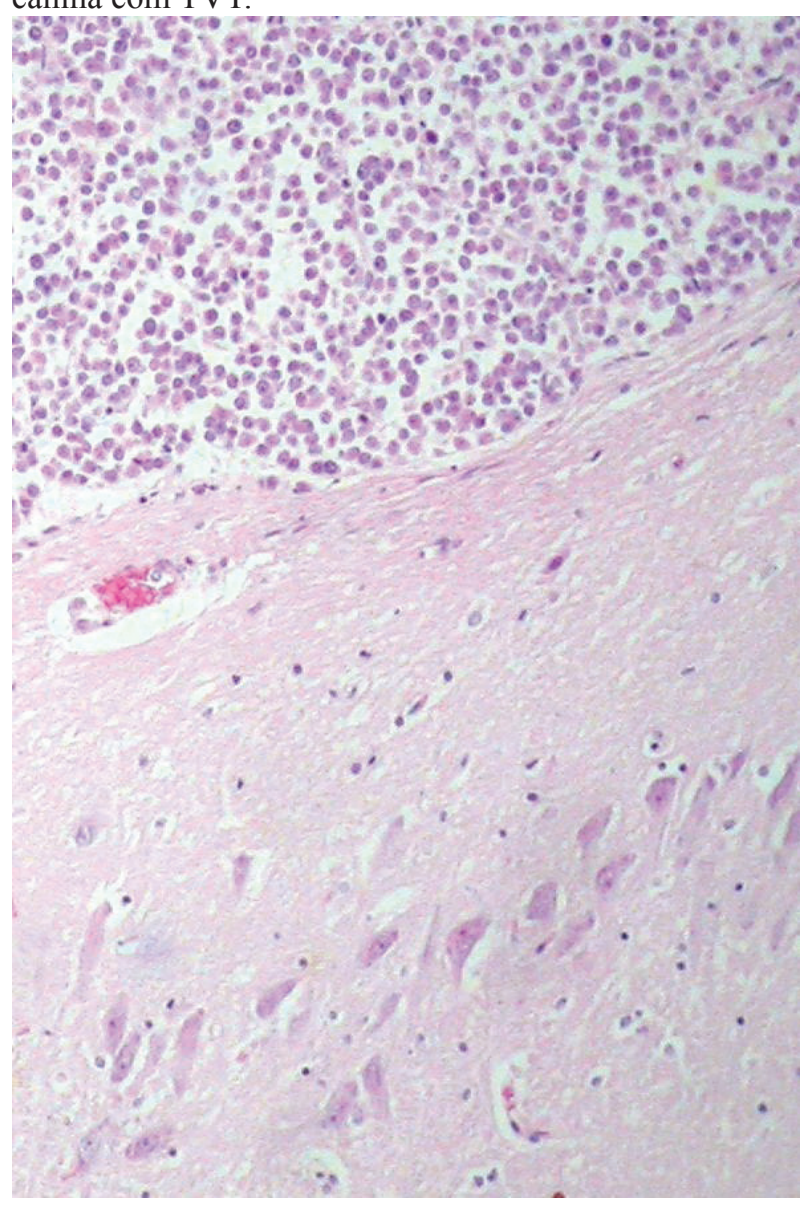

Fonte: Elaboração dos autores.

\section{Discussão}

O TVT encontrado no paciente foi classificado como padrão plasmocitóide, devidoa maioria das células apresentarem aspecto semelhante à plasmócitos (AMARAL et al., 2007). De acordo com alguns autores, o TVT neste padrão em localizações extragenitais, apresenta-se com maior malignidade e com respostas deficientes ao tratamento aos quimioterápicos (AMARAL et al., 2007; FILGUEIRA, 2010).

As massas pulmonares detectadas na necropsia não foram observadas durante a avaliação radiológica do paciente devido, provavelmente, terem sido desenvolvidas após o exame. Fato que deve ter ocorrido devido à malignidade do tumor, associado à imunossupressão e a perda de peso do paciente, culminando com o desenvolvimento de metástase ocular, pulmonar e no sistema nervoso central. De acordo com a literatura, a disseminação do tumor ocorre em condições de desnutrição e/ou imunossupressão, permitindo a migração de células de TVT via corrente sanguínea e linfática com a instalação em diferentes tecidos (RODRIGUES; ALESSI; LAUS, 2001; PARK et al., 2006; SILVA et al., 2007). Tem sido documentada resistência à vincristina no tratamento de TVT, fator significativo ao aumento da incidência nos últimos anos (DALECK et al., 2008; GARCEZ et al., 2010).

O desenvolvimento de células de TVT em regiões do tálamo e colículos encefálicos, possivelmente tenham ocorrido devido aos quimioterápicos utilizados não apresentarem capacidade para atravessar a barreira hematoencefálica (CHABNER et al., 2001). Conforme Whortman (1956), a disseminação de TVT ao SNC pode ocorrer através dos seios venosos, que proporcionam uma possível via para o tumor chegar ao cérebro, a partir de sítios primários na cavidade abdominal e pélvica. Portanto, é necessário investigação de TVT no sistema nervoso central, em pacientes com tumor e que apresentam sinais neurológicos.

\section{Conclusões}

Este relato registra o primeiro caso no Brasil de tumor venéreo transmissível vaginal com metástase para o sistema nervoso central, além do envolvimento do tecido cutâneo, ocular e pulmonar, e descreve a resistência do TVT ao tratamento com vincristina.

\section{Agradecimentos}

CAPES, CNPq e FAPERGS. Ao Hospital de Clínicas Veterinária (HCV) e Laboratório Regional 
de Diagnóstico (LRD) da Universidade Federal de Pelotas (UFPel).

\section{Referências}

AMARAL, A. S.; SILVA, S. B.; FERREIRA, I.; FONSECA, L. S.; ANDRADE, F. H. E.; GASPAR, L. F. J.; ROCHA, N. S. Cytomorphological characterization of transmissible canine venereal. Revista Portuguesa de Ciências Veterinárias, Lisboa, v. 102, n. 563-564, p. 253260, 2007.

CHABNER, B.A.; RYAN, D. P.; PAZ-ARES, L.; GARCIACARBONERO, R.; CALABRESI, P. Antineoplasticagents. In: HARDMAN, J. G.; LIMBIRD, L. E.; GILMAN, A. The pharmacological basis of therapeutics. 10. ed. New York: MC GRAW- HILL, 2001. p. 1385-1459.

DALECK, C. R.; SILVA, M. C. V., DE NARDI, A. B.; FERNANDES, S.; CASTRO, J. Utilização da doxorrubicina e ciclofosfamida no tratamento do tumor venéreo transmissível canino intranasal: relato de caso. $A$ Hora Veterinária, Porto Alegre, v. 164, n. 28, p. 58-61, 2008.

FERREIRA, A. J. A.; JAGGY, A.; VAREJÃO, A. P.; FERREIRA, M. L. P.; CORREIA, J. M. J.; MULAS, J. M.; ALMEIDA, O.; OLIVEIRA, P.; PRADA, J. Brain and ocular metástases from a transmissible veneral tumour in a dog. Journal of Small Animal Practice, Malden, v. 41, n. 4, p. 165-168, 2000.

FILGUEIRA, K. D. Tumor venéreo transmissível canino com localização primária e única em cavidade oral. Acta Scientiae Veterinariae, Porto Alegre, v. 38, n. 1, p. 91-94, 2010.
GARCEZ, T. N. A.; GOMES, C.; MÖSCHBÄCHER, P. D.; OLIVEIRA, L. O.; CONTESINI, E. A. Tratamento de tumor venéreo transmissível extragenital resistente à vincristina: quimioterapia antineoplásica e cirurgia reconstrutiva. MedVep, Curitiba, v. 8, n. 25, p. 304-307, 2010.

PARK, M.; KIM, Y.; KANG, M.; OH, S.; CHO, D.; SHIN, N.; KIM, D. Disseminated transmissible venereal tumor in a dog. Journal of Veterinary Diagnostic Investigation, Georgia, v. 18, n. 1, p. 130-133, 2006.

RODRIGUES, G. N.; ALESSI, A. C.; LAUS, J. L. Tumor venéreo transmissível intra-ocular em cão. Ciência Rural, Santa Maria, v. 31, n. 1, p. 141-143, 2001.

SCARPELLI, K.; VALLADÃO, M. L.; METZE, K. Predictive factors for the regression of canine transmissible venereal tumor during vincristine therapy. Journal Veterinary, England, v. 183, n. 3, p. 362-363, 2010.

SILVA, M. V. S.; BARBOSA, R. R.; SANTOS, R. C.; CHAGAS, R. S. N.; COSTA, W. P. Avaliação epidemiológica e diagnóstica e terapêutica do tumor venéreo transmissível (TVT) na população canina atendida no Hospital Veterinário da UFERSA. Acta Veterinaria Brasilica, Mossoró, v. 1, n. 1, p. 28-32, 2007.

WHORTMAN, P. The longitudinal vertebral venous sinuses of the dog. Functional aspects. American Journal of Veterinary Research, Ilinois, v. 17, n. 64, p. 349-363, 1956. 
